\title{
A gallery of cool hypergiants - imaging their circumstellar environments
}

\author{
Michael T. Schuster, Roberta M. Humphreys, Nathan Smith, \\ Kris Davidson, and Robert D. Gehrz \\ Department of Astronomy, University of Minnesota, \\ 116 Church Street SE, Minneapolis, MN55455, USA
}

\begin{abstract}
High resolution imaging with HST-wFPC2 of seven of the mast luminous evolved cool stars shows that the three OH/IR stars NML Cyg, VX Sgr and S Per have circumstellar nebulae while no ejecta was detected for the M-type supergiant $\mu$ Cep and the yellow hypergiants $\rho$ Cas, HR 8752 and HR 5171a.
\end{abstract}

Cool hypergiants are highly unstable, evolved massive stars that lie just below the upper luminosity boundary in the H-R diagram with apparent temperatures $\lesssim 10000 \mathrm{~K}$ (spectral types $\mathrm{A}$ to $\mathrm{M}$ ). Observations of two of these stars, IRC+10420 (Humphreys et al. 1997, 2002) and VY CMa (Smith et al. 2001) have revealed very complex circumstellar environments with multiple arcs, rings, knots and jet-like features imbedded in more uniform, diffuse nebulae. We have obtained high-resolution, multi-wavelength images of seven additional very luminous cool stars, to better understand their mass loss histories and to sample them at a variety of stages in their brief evolution as cool hypergiants. Their positions on the H-R diagram is shown in Figure 1.

The OH/IR M-type supergiants NML Cyg, VX Sgr, and S Per, the normal M-type supergiant $\mu$ Cep, and the 'yellow' hypergiants $\rho$ Cas, HR 8752 and HR 5171a were imaged with HST-WFPC2 . The images were all processed with the standard routines in IRAF/STSDAS with the most recent reference files. The PSF-patched long exposures were drizzled to maximize the spatial resolution.

The powerful OH/IR source NML Cyg has a very obvious circumstellar nebula $\sim 500 \times 1000 \mathrm{AU}$ with a peculiar asymmetric bean shape, which may be due to interaction with the UV radiation and winds from the nearby Cyg OB2 association. The shape is similar to the theoretical contours calculated by Morris \& Jura (1983) for the associated H II region, but on a much smaller scale. The equally well-studied $\mathrm{OH} / \mathrm{IR}$ source VX Sgr is non-stellar with an image equal to HST's diffraction limit at $0.5 \mu \mathrm{m}$, implying a size of $\sim 100 \mathrm{AU}$ for its extended envelope. S Per has an elongated circumstellar envelope $\sim 800 \mathrm{AU}$ across which unlike NML Cyg and VX Sgr does not obscure the star. However, we find no evidence for extended structure for $\rho$ Cas, HR 8752, HR 5171a and $\mu$ Cep within 0 ' 1 of the stars and down to the WFPC2 read noise $\sim 2^{\prime \prime}$ away.

Cornelis de Jager (1998) has suggested that very massive stars evolving blueward encounter a region of increasing dynamical instability, that he calls the 'yellow void'. He specifically suggests that $\rho$ Cas, HR 8752 and IRC+10 420 are post-red supergiants and that all of the yellow hypergiants may be in a post- 


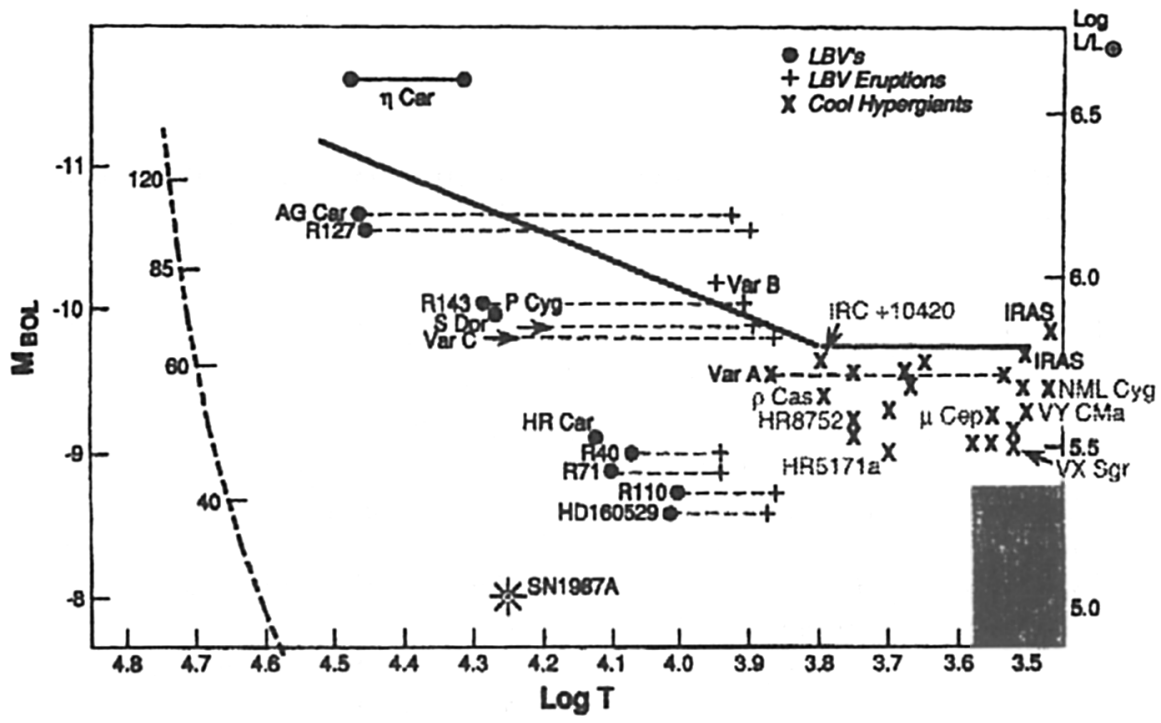

Figure 1. A schematic H-R diagram showing the location of the Luminous Blue Variables and the cool hypergiants. The stars we observed are shown in gray. The solid line is the empirical upper luminosity boundary.

RSG stage, which is confirmed for IRC+10 420 (Jones et al. 1993; Oudmaijer et al. 1996; Humphreys et al. 1997). If $\rho$ Cas and HR 8752 have been RSGs, then we would expect to find distant fossil shells from that high mass-losing stage, and nearby material from more recent mass loss episodes as yellow hypergiants. At this time, our results suggest that these stars are stellar. There is no evidence for nebulosity either near the stars or more distant.

Acknowledgments. Support for this study was provided by NASA through grant number GO-8099 from the Space Telescope Science Institute, which is operated by the Association of Universities for Research in Astronomy, Inc., under NASA contract NAS 5-26555.

\section{References}

de Jager, C. 1998, The A\&A Review 8, 145

Humphreys, R.M., Davidson, K., Smith, N. 2002, AJ 124, 1026

Humphreys, R.M., Smith, N., Davidson, K., Jones, T.J., et al. 1997, AJ 114, 2778

Jones, T.J., Humphreys, R.M., Gehrz, R.D., et al. 1993, ApJ 411, 323

Morris, M., Jura, M. 1983, ApJ 267, 179

Oudmaijer, R.D., Groenewegen, M.A.T., Matthews, H.E., Blommaert, J.A.D., Sahu, K.C. 1996, MNRAS 280, 1062

Smith, N., Humphreys, R.M., Davidson, K., Gehrz, R.D., Schuster, M.T., Krautter, J. 2001, AJ 121, 1111 